Published in final edited form as:

Clin Breast Cancer. 2017 February ; 17(1): 23-28. doi:10.1016/j.clbc.2016.07.002.

\title{
Characteristics and outcomes of breast cancer patients with leptomeningeal metastasis
}

\author{
Aki Morikawa, MD,PhD ${ }^{1,}$, Lilly Jordan, BA ${ }^{1}$, Raquel Rozner, BS ${ }^{1}$, Sujata Patil, PhD $^{3}$, \\ Adrienne Boire, MD, $\mathrm{PhD}^{2}$, Elena Pentsova, $\mathbf{M D}^{2}$, and Andrew D. Seidman, MD ${ }^{1}$ \\ ${ }^{1}$ Breast Cancer Medicine Service, Memorial Sloan Kettering Cancer Center, 300 E. $66^{\text {th }}$ Street, \\ New York, NY 10065 \\ 2Department of Neurology, Memorial Sloan Kettering Cancer Center, 1275 York Avenue, New \\ York, NY 10065 \\ ${ }^{3}$ Department of Biostatistics, Memorial Sloan Kettering Cancer Center, 1275 York Avenue, New \\ York, NY 10065
}

\begin{abstract}
Background-Disease presentation, prognostic factors, and treatment patterns for breast cancer patients with leptomeningeal metastasis are not well-characterized. In this study, we examined patient characteristics and prognostic factors for survival after a diagnosis of leptomeningeal metastasis.
\end{abstract}

\begin{abstract}
Patients and Methods-318 consecutive breast cancer patients diagnosed with leptomeningeal metastasis from January 1998 to December 2013 at Memorial Sloan Kettering Cancer Center were identified. Clinico-pathologic and treatment information were obtained by retrospective review. Associations with time from leptomeningeal diagnosis to death were evaluated by Kaplan-Meier curves, log-rank tests, and Cox proportional hazard models.
\end{abstract}

Results-Of the 318 patients, $44 \%$ were HR+HER2-,18\% HR+HER2+, $8.5 \%$ HR-HER2+, and $25.5 \%$ triple negative; $4 \%$ had missing information. The median survival was 3.5 months (95\% CI: 3.0, 4.0) with 63 patients (20\%) surviving greater than 1 year. Recent diagnosis (after 2006), HER2+ subtype, higher performance status, cranial only involvement, and no evidence of non-

\footnotetext{
Corresponding author: Andrew D. Seidman, MD, Evelyn Lauder Breast Center, Memorial Sloan Kettering Cancer Center, 300 East $66^{\text {th }}$ Street, New York, NY 10065, 646-888-4559 (phone), 646-888-4917 (fax), seidmana@mskcc.org.

Current affiliation: Division of Hematology/Oncology, Department of Medicine, University of Michigan, Ann Arbor, MI 48109. Aki Morikawa, morikawa@med.umich.edu, University of Michigan

Lilly Jordan, ljordan@med.wayne.edu, Memorial Sloan Kettering Cancer Center

Raquel Rozner, raquelrozner@gmail.com, Memorial Sloan Kettering Cancer Center

Sujata Patil, patils@mskcc.org, Memorial Sloan Kettering Cancer Center

Adrienne Boire, boirea@mskcc.org, Memorial Sloan Kettering Cancer Center

Elena Pentsova, pentove@mskcc.org, Memorial Sloan Kettering Cancer Center

Andrew D. Seidman, seidmana@mskcc.org, Memorial Sloan Kettering Cancer Center

Publisher's Disclaimer: This is a PDF file of an unedited manuscript that has been accepted for publication. As a service to our customers we are providing this early version of the manuscript. The manuscript will undergo copyediting, typesetting, and review of the resulting proof before it is published in its final citable form. Please note that during the production process errors may be discovered which could affect the content, and all legal disclaimers that apply to the journal pertain.

Potential conflict of interest: Principal investigator for clinical trial NCT02650752 (AS), Site principal investigator for clinical trial NCT01325207 (EP), Site principal investigator for clinical trial NCT02308020 (AM).
} 
central nervous system disease were independently associated with improved survival in multivariate analysis.

Conclusions-Despite the improvement noted with more recent year of diagnosis, survival following a diagnosis of leptomeningeal metastasis remains poor. Similar to patients with parenchymal brain metastasis only, the survival differs among difference receptor subtypes. A closer examination to identify factors, such as introduction of new systemic therapies that may contribute to longer-term survival may provide insight to improve management of these patients. In addition, factors we identified that are associated with survival may be considered as stratification variables in the design of future randomized clinical trials in this population.

\section{MicroAbstract}

A retrospective cohort study of 318 breast cancer patients with leptomeningeal metastases was conducted to describe patient characteristics and outcomes.. Independent factors associated with survival after the diagnosis of leptomeningeal metastases were identified. Such information is useful in management and in clinical trial development of breast cancer patients with leptomeningeal metastases.

\section{Keywords}

breast cancer; leptomeningeal disease; prognosis

\section{Introduction}

Breast cancer is one of the common solid tumors to metastasize to the central nervous system (CNS). In particular, leptomeningeal metastasis (LM) is associated with significant morbidity and mortality. The incidence of LM in breast cancer is reported to be approximately 5\% [1]. Additionally, we reported a 14\% prevalence of concurrent LM at the time of brain metastasis diagnosis in our HER2 positive breast cancer brain metastases cohort [2].

The survival of patients with LM is known to be very short and therapeutic options and their efficacy are often limited [1,3]. Current treatment options for LM are local therapies such as radiotherapy and intrathecal/intraventricular therapy. However, the efficacy of intrathecal/ intraventricular therapy is not well established in breast cancer patients and associated with significant neurotoxicity [4]. Therefore, assessing appropriate candidates for active therapeutic intervention is an important consideration. Among breast cancer patients with CNS metastases, the HER2+ subpopulation has a relatively better prognosis and in this group CNS-penetrating targeted therapy is under investigation.

For patients with LM, disease presentation, prognostic factors, and treatment patterns are not well-defined. Improvement in breast cancer treatment including HER2-targeted therapy, may have improved the outcome of patients with LM over time. We hypothesize that receptor subytpe may be an important prognostic factor for LM patients, similarly to what has been reported for patients with brain metastases [5-7]. 
The objectives of this retrospective study were to examine a modern cohort of breast cancer patients diagnosed with leptomeningeal disease at Memorial Sloan Kettering Cancer Center (MSKCC), describe patient characteristics and treatment patterns, and determine factors associated with survival after LM diagnosis. The results of this study will add to our understanding of the contemporary epidemiology of LM in breast cancer and provide prognostic factors which will be useful in management of LM patients and in designing future clinical trials in this population.

\section{Patients and Methods}

\section{Study population}

Consecutive breast cancer patients diagnosed with LM from January 1998 to December 2013 and treated at MSKCC were identified from an institutional database after approval from the Institutional Review Board. LM diagnosis was determined based on MRI image reports or positive cerebrospinal fluid (CSF) studies. Additionally, patients with suspicious LM on MRI or suspicious CSF cytology were included if the treating oncologist or neurologist documented the LM diagnosis and LM-directed therapy (e.g. intrathecal therapy).

\section{Data collection}

Clinico-pathologic information was abstracted by retrospective chart review and included the following variables: hormone receptor (HR, estrogen and progesterone) status, HER2 status, stage, and grade of the primary tumor, age at LM diagnosis, date of initial LM diagnosis, site of LM involvement (classified as cranial only, spinal only, or both), method of LM diagnosis (cytology, imaging, or both), metastatic diagnosis (date of metastatic breast cancer diagnosis) and extent (sites of metastatic disease, e.g. liver, bone, lung) and control of non-CNS disease at LM diagnosis (classified either progression, non-progression, or no evidence of disease), history of brain metastasis diagnosis, Karnofsky Performance Score (KPS) at LM diagnosis, local CNS directed therapy (radiation, surgery, and intrathecal therapy) and systemic therapy. KPS was dichotomized at the cut off of 70, and the date of initial LM diagnosis was dichotomized at 2006, a midpoint between1998 - 2013, for further analyses.

\section{Statistical analysis}

Clinico-pathologic data was summarized using median and ranges for continuous variables and proportions for categorical variables. Overall survival was defined as the time from LM diagnosis to death or last date of follow-up. Survival estimates were calculated by KaplanMeier method and comparisons were made using the log rank test. In addition, hazard ratios were calculated using a Cox proportional hazards model. Multivariable Cox regression analysis was performed to identify prognostic factors for overall survival. Proportional hazard assumptions were evaluated for each variable considered in the multivariable analysis. Selection of variables for the multivariable model was based on the significant of univariate findings. Variables known to be prognostic (such as age) and thus important to adjust for in the model were also included. All the statistical calculations were performed 
using SAS version 9.4 and R. P-values less than .05 were considered to be statistically significant and all tests were two-sided.

\section{Results}

\section{Patient characteristics}

A total of 318 breast cancer patients with LM that met the study inclusion criteria were identified from January 1, 1998 to December 31, 2013. The patient characteristics and treatment information are described in Table 1. The HR+HER2- subtype was the most common: 44\% HR+HER2-, 26\% HR+/-HER2+, and 26\% HR-HER2- (4\% with missing information on ER, PR or HER2 status). 95\% (303/318) of the cohort had MRI or CT imaging consistent with LM. 44\% (141/318) of our cohort had CSF cytology evaluation, and $34 \%$ of the cohort had positive (100/318) or suspicious (7/318) CSF cytology. In our cohort, the LM treatments received included radiation, ventriculoperitoneal shunt placement, intrathecal/intraventricular therapy, and systemic therapy (e.g. intravenous high dose chemotherapy).

\section{Survival from LM diagnosis}

The median time to death for the cohort was 3.5 months (95\% CI: 3.0, 4.0) (Figure 1a). The HR-HER2- subgroup had the worst median survival from LM diagnosis of 2.5 months (95\% CI: 2.0, 3.1) compared to the other subtypes: HR+HER2- 3.9 months (95\% CI: 3.0, 5.7) and HR+/- HER2+ 5.2 months (95\%CI: 3.2, 7.8) (Figure 1b). We hypothesized that with increasing use of anti-HER2 therapies and the introduction of lapatinib (a small molecule tyrosine kinase inhibitor anti-HER2 therapy), we might observe a change in survival for HER2+ patients over time. We dichotomized the cohort and examined the median survivals for HER2+ patients. In an exploratory analysis, the median survival was 3.3 months (95\% CI: 2.4, 5.0) prior to the year 2005 and 7.0 months (95\%CI: 3.7, 12.2) thereafter. The final multivariable model included age at LM diagnosis, year of LM diagnosis, KPS, receptor subtype, site of LM involvement (e.g. cranial, spinal, or both), and non-CNS disease control status (Table 2). As expected, patients with poorer KPS $(\leq 70)$ had a shorter survival (HR $2.3195 \%$ CI: 1.64, 3.24, $\mathrm{p}=<0.001$ ). The year of LM diagnosis (dichotomized at year 2005) remained a significant factor in a multivariate analysis (HR $1.40,95 \%$ CI:1.04, 1.88, $\mathrm{p}=0.03$ ).

Unlike patients with brain metastasis, where HER2 expression is associated with a longer survival, in our LM cohort, there was no statistically significant difference in overall survival between the HER2+ and HR+HER2- patients (HR 1.01, 95\%CI:0.72, 1.420, $\mathrm{p}=0.95$ ). However, as expected HR-HER2 - patients had a worse survival compared to the HR +HER2- patients (HR 1.76, 95\% CI:1.26, 2.46, p <0.01). Interestingly, the sites of LM involvement remained a significant factor where cranial only involvement was favorable as opposed to spinal involvement (type III p-value 0.04).

\section{Discussion}

This is the largest cohort of breast cancer patients from a single center with LM diagnosis reported to date $[8,9]$. Consistent with other reports, we have observed that patients 
diagnosed with LM display a short median survival after LM diagnosis: the majority of these succumb to the disease within a year. Importantly, 20\% (63/318) of our cohort survived longer than a year. We therefore examined prognostic factors associated with survival after LM diagnosis. Because there is a major selection bias associated with LM therapy administration (e.g. systemic and intrathecal/intraventricular therapy), the use of LM therapy was evaluated in a descriptive manner only and was not examined as a possible prognostic factor in our model.

Receptor subtype has proved to be an important prognostic factor for breast cancer patients with parenchymal brain metastasis $[10,11]$. We therefore hypothesized that receptor subtype would be a similarly important prognostic factor as observed for breast cancer patients with LM. HER2+ patients with brain metastasis have a favorable prognosis compared to other subtypes [12, 13]. However, in our cohort, survival from LM diagnosis was similar for HER2+ and HR+HER2- patients. Consistent with other studies of LM, triple negative (HR -HER2-) patients had the shortest survival after diagnosis [9, 14].

In patients with parenchymal brain metastasis, it has been theorized that the relatively favorable prognosis associated with HER2+ CNS metastasis is due to the improvement in non-CNS disease control from the use of anti-HER2 drugs and the introduction of lapatinib, which has demonstrated CNS uptake and activity in CNS metastasis[15-20]. In an exploratory univariate analysis, we observed that the survival for HER2+ patients who were diagnosed in more recent years (after 2005, coinciding with increasing use of lapatinib for HER2+ CNS metastases at our institution) had a longer median survival compared to those who were diagnosed prior to 2005. However, due to the small number of patients, an interaction between the subtype and the year of LM diagnosis was not examined in our multivariate model.

No effective standard targeted therapy for LM has been introduced for routine clinical practice during the study period [21]. We therefore hypothesized that the improvement in survival over time might be attributable to improvement in systemic therapy. With advent of small molecule drugs and drugs modified to improve CNS penetration, systemic therapy for CNS metastasis is now actively investigated in prospective clinical trials [15]. The efficacy of systemic therapy for LM may therefore be best examined through prospective clinical trials specifically targeting this population.

Intrathecal chemotherapy for breast cancer patients remains controversial [4]. First, therapies directly administered into this space have limited penetration, so intrathecal therapy is generally reserved for patients with either MRI-negative or non-bulky leptomeningeal metastases. Secondly, a limited range of drugs are classically suitable for IT administration (methotrexate, cytarabine, and thiotepa). Attempts are underway to expand the repertoire of IT therapeutics in breast cancer. Current studies on IT Herceptin for patients with Her 2 positive breast cancer leptomeningeal metastasis are ongoing; results are expected soon.

Unexpectedly, we observed that patients with cranial-only involvement of LM demonstrated improved survival. It is unclear if this finding is due to a difference in biology or detection bias, as most but not all patients underwent both brain and total spine imaging at the time of 
LM diagnosis. It is possible that the patients with spinal LM involvement may have had a delay in diagnosis as neurologic symptoms referable to the spine and spinal roots may have been misattributed to bone metastasis and other non-LM diagnoses. In an exploratory analysis, we examined specific sites of non-CNS metastases (e.g. lung, bone, liver) as variables, but the presence of bone metastasis was not a significantly associated with outcome in univariate analysis. Our observation has an important implication for considering routine and systematic imaging of both cranial and spinal compartments for patients with LM [22]. It would be of interest to systematically evaluate the extent of LM involvement in a prospective manner to validate whether the site of LM involvement is an important prognostic factor.

Our study has several limitations due to its retrospective nature. Reflective of current clinical practice, not all patients underwent CSF cytologic evaluation and complete imaging of the neuro-axis. However, we conducted an exploratory analysis according to the LM diagnosis method. The survival did not differ between patients who had positive CSF cytology and patients whose LM diagnosis was made with image findings only (without CSF evaluation). The patients who were diagnosed with image finding but with negative cytology had a significantly longer survival $(\mathrm{p}=0.01)$. Such difference may be explained by the disease burden or possible misclassification. We did include three patients for whom we were unable to obtain source information on imaging or CSF cytology as those evaluations were reported from an outside institution's records, and the patients were treated clinically for LM. . Receipt of systemic therapy or LM therapy was not evaluated as an independent prognostic variable given that it would have been influenced by the performance status and the status and extent of non-CNS disease.

\section{Conclusions}

The majority of LM patients have a limited survival, but a subset of patients with a more favorable prognosis exists for whom further active anti-cancer therapy may be appropriate. Receptor subtype is an important prognostic factor and should be accounted for in future studies involving breast cancer patients with LM. An improvement in outcomes for HER2+ patients with LM parallels the emergence of highly effective anti-HER2 therapy, and suggests a potential treatment effect. Several trials are currently evaluating molecular targeted drug therapy (e.g. high dose lapatinib, intrathecal trastuzumab, and abemaciclib) for breast cancer patient with LM (NCT02650752, NCT01325207, NCT02308020). Our data supports the ongoing clinical trials examining the use of systemic drugs to improve the outcome of the LM patients.

\section{Acknowledgments}

Funding/support: Judah Gubbay Memorial Fund

Andrew D. Seidman, the corresponding author, had full access to all of the data in the study and takes responsibility for the integrity of the data and the accuracy of the data analysis.

\section{Abbreviations}

LM leptomeningeal metastasis 
BC breast cancer

HR hormone receptor

CNS central nervous system

KPS Karnofsky Performance Score

OS overall survival

\section{References}

1. Grossman SA, Krabak MJ. Leptomeningeal carcinomatosis. Cancer Treat Rev. 1999; 25:103-119. [PubMed: 10395835]

2. Morikawa, A., Diab, A., Patil, S., et al. San Antonio Breast Cancer Symposium. San Antonio, TX: Cancer Research; 2013. Patient characteristics and outcomes for patients with HER2-overexpressing breast cancer with brain metastases undergoing radiation therapy in the pre- and post adjuvant trastuzumab era. P6-11-02

3. Le Rhun E, Taillibert S, Zairi F, et al. A retrospective case series of 103 consecutive patients with leptomeningeal metastasis and breast cancer. J Neurooncol. 2013; 113:83-92. [PubMed: 23456656]

4. Boogerd W, van den Bent MJ, Koehler PJ, et al. The relevance of intraventricular chemotherapy for leptomeningeal metastasis in breast cancer: a randomised study. Eur J Cancer. 2004; 40:2726-2733. [PubMed: 15571954]

5. Morikawa, A., Diab, A., Patil, S., et al. 2013 Breast Cancer Symposium. San Francisco, USA: J Clin Oncol; 2013. Radiation therapy for breast cancer with central nervous system metastases: A contemporary experience at Memorial Sloan-Kettering Cancer Center.

6. Sperduto PW, Kased N, Roberge D, et al. Effect of tumor subtype on survival and the graded prognostic assessment for patients with breast cancer and brain metastases. Int J Radiat Oncol Biol Phys. 2012; 82:2111-2117. [PubMed: 21497451]

7. Subbiah IM, Lei X, Weinberg JS, et al. Validation and Development of a Modified Breast Graded Prognostic Assessment As a Tool for Survival in Patients With Breast Cancer and Brain Metastases. J Clin Oncol. 2015; 33:2239-2245. [PubMed: 25987700]

8. Hyun JW, Jeong IH, Joung A, et al. Leptomeningeal metastasis: Clinical experience of 519 cases. Eur J Cancer. 2016; 56:107-114. [PubMed: 26841095]

9. Abouharb S, Ensor J, Loghin ME, et al. Leptomeningeal disease and breast cancer: the importance of tumor subtype. Breast Cancer Res Treat. 2014; 146:477-486. [PubMed: 25038877]

10. Sperduto PW, Kased N, Roberge D, et al. The effect of tumor subtype on the time from primary diagnosis to development of brain metastases and survival in patients with breast cancer. $\mathrm{J}$ Neurooncol. 2013

11. Yang TJ, Oh JH, Folkert MR, et al. Outcomes and prognostic factors in women with 1 to 3 breast cancer brain metastases treated with definitive stereotactic radiosurgery. Int J Radiat Oncol Biol Phys. 2014; 90:518-525. [PubMed: 25194669]

12. Sperduto PW, Kased N, Roberge D, et al. The effect of tumor subtype on the time from primary diagnosis to development of brain metastases and survival in patients with breast cancer. $\mathrm{J}$ Neurooncol. 2013; 112:467-472. [PubMed: 23462853]

13. Yang TJ, Oh JH, Folkert MR, et al. Outcomes and Prognostic Factors in Women With 1 to 3 Breast Cancer Brain Metastases Treated With Definitive Stereotactic Radiosurgery. International Journal of Radiation Oncology Biology Physics. 2014; 90:518-525.

14. Yust-Katz S, Garciarena P, Liu D, et al. Breast cancer and leptomeningeal disease (LMD): hormone receptor status influences time to development of LMD and survival from LMD diagnosis. J Neurooncol. 2013; 114:229-235. [PubMed: 23756727]

15. Lin NU. Breast cancer brain metastases: new directions in systemic therapy. Ecancermedicalscience. 2013; 7:307. [PubMed: 23662165] 
16. Lin NU, Carey LA, Liu MC, et al. Phase II trial of lapatinib for brain metastases in patients with human epidermal growth factor receptor 2-positive breast cancer. J Clin Oncol. 2008; 26:19931999. [PubMed: 18421051]

17. Lin NU, Dieras V, Paul D, et al. Multicenter phase II study of lapatinib in patients with brain metastases from HER2-positive breast cancer. Clin Cancer Res. 2009; 15:1452-1459. [PubMed: 19228746]

18. Lin NU, Eierman W, Greil R, et al. Randomized phase II study of lapatinib plus capecitabine or lapatinib plus topotecan for patients with HER2-positive breast cancer brain metastases. J Neurooncol. 2011; 105:613-620. [PubMed: 21706359]

19. Morikawa A, Peereboom DM, Thorsheim HR, et al. Capecitabine and lapatinib uptake in surgically resected brain metastases from metastatic breast cancer patients: a prospective study. Neuro Oncol. 2015; 17:289-295. [PubMed: 25015089]

20. Saleem A, Searle GE, Kenny LM, et al. Lapatinib access into normal brain and brain metastases in patients with Her-2 overexpressing breast cancer. EJNMMI Res. 2015; 5:30. [PubMed: 25977884]

21. Chamberlain M, Soffietti R, Raizer J, et al. Leptomeningeal metastasis: a Response Assessment in Neuro-Oncology critical review of endpoints and response criteria of published randomized clinical trials. Neuro Oncol. 2014; 16:1176-1185. [PubMed: 24867803]

22. Clarke JL, Perez HR, Jacks LM, et al. Leptomeningeal metastases in the MRI era. Neurology. 2010; 74:1449-1454. [PubMed: 20439847] 


\section{Clinical Practice Points}

There is a paucity of information regarding patient characteristics and prognosis of breast cancer patients with leptomeningeal disease, especially in context of changes in therapeutic options and epidemiology of CNS metastasis in breast cancer patients over the recent decades. Our study showed that breast cancer patients who are more recently diagnosed with leptomeningeal metastases and HER2 positive patients had a longer survival. Despite fact that leptomeningeal metastasis may be associated with a limited survival, there is a subset of patients who may benefit from continued active therapy as demonstrated by $20 \%$ of our cohort surviving longer than one year. The study informs ongoing efforts to study new therapeutic options for leptomeningeal patients. Those patients with good prognostic features may be reasonable candidates for additional active therapies and leptomeningeal metastasis focused clinical trials. 
A

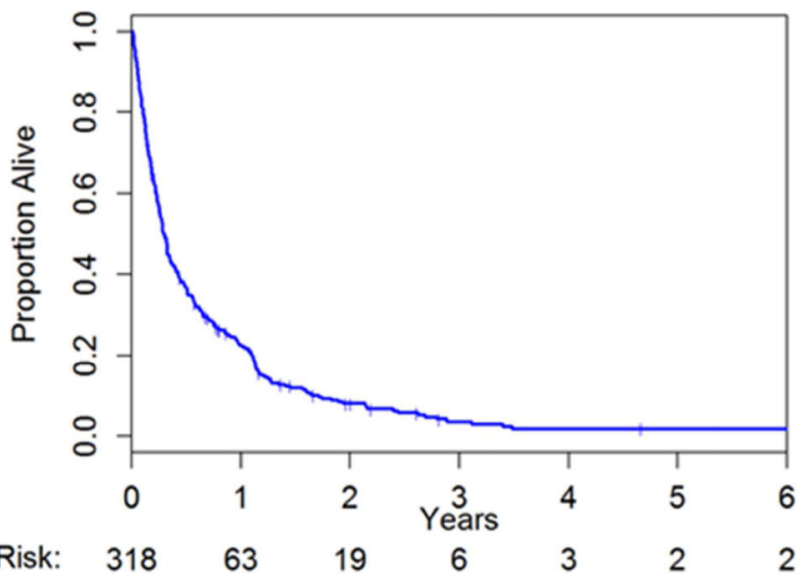

B

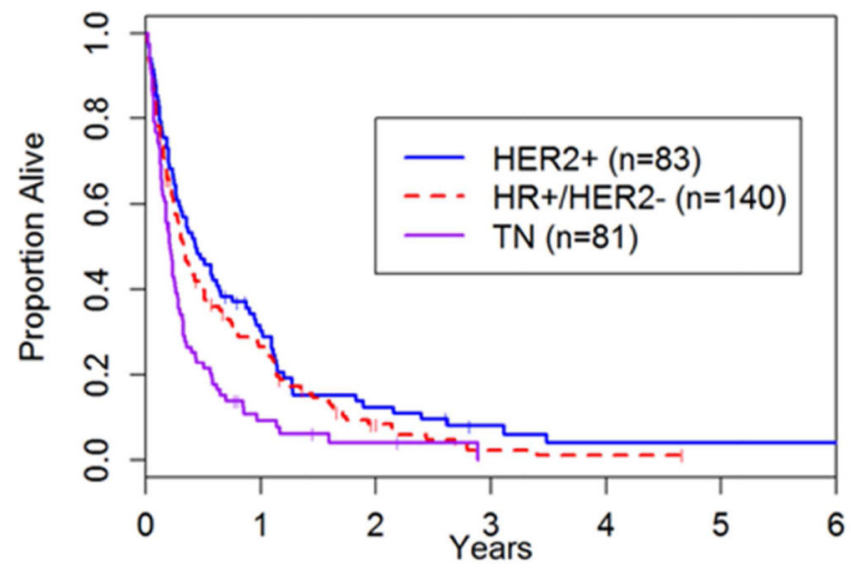

Figure 1.

Overall survival from diagnosis of leptomeningeal disease. (A) Overall cohort of 318 patients; (B) By three receptor subtypes; 
Table 1

Patient characteristics $(\mathrm{N}=318)$

\begin{tabular}{|c|c|c|c|c|}
\hline Characteristics & & $\mathbf{N}$ & $\%$ & $\begin{array}{c}\text { Univariate } \\
\text { association } \\
\text { with OS }\end{array}$ \\
\hline \multirow{2}{*}{ Age at LM diagnosis (years) } & Median & \multicolumn{2}{|c|}{54} & \\
\hline & Range & \multicolumn{2}{|c|}{$23-84$} & \\
\hline \multirow{2}{*}{ Age at BC diagnosis (years) } & Median & \multicolumn{2}{|c|}{46.4} & \\
\hline & Range & \multicolumn{2}{|c|}{$20.5-77.4$} & \\
\hline \multirow{2}{*}{$\begin{array}{l}\text { Time from MBC to LM } \\
\text { diagnosis (months) }\end{array}$} & Median & \multicolumn{2}{|c|}{22.6} & \\
\hline & Range & \multicolumn{2}{|c|}{$0-238$} & \\
\hline \multirow{2}{*}{ Year of LM diagnosis } & $1998-2005$ & 98 & $31 \%$ & $<0.01$ \\
\hline & $2006-2013$ & 220 & $69 \%$ & \\
\hline \multirow{4}{*}{ Receptor status } & HR+/HER2- & 140 & $44 \%$ & $<0.01$ \\
\hline & HR any/ HER2+ & 83 & $26 \%$ & \\
\hline & HR- / HER2- & 81 & $26 \%$ & \\
\hline & Missing & 14 & $4 \%$ & \\
\hline \multirow{3}{*}{ KPS at LM diagnosis } & $<70$ & 58 & $18 \%$ & $<0.01$ \\
\hline & $\geq 70$ & 223 & $70 \%$ & \\
\hline & Missing & 37 & $12 \%$ & \\
\hline \multirow{5}{*}{$\begin{array}{l}\text { Stage at the time of initial BC } \\
\text { diagnosis }\end{array}$} & I & 26 & $8 \%$ & \\
\hline & II & 68 & $21 \%$ & \\
\hline & III & 40 & $13 \%$ & \\
\hline & IV & 78 & $25 \%$ & \\
\hline & Missing & 106 & $33 \%$ & \\
\hline \multirow{3}{*}{ Grade of primary BC } & $\mathrm{I} / \mathrm{II}$ & 36 & $11 \%$ & 0.06 \\
\hline & III & 199 & $63 \%$ & \\
\hline & Missing & 83 & $26 \%$ & \\
\hline \multirow{4}{*}{$\begin{array}{l}\text { Method of LM diagnosis } \\
\text { confirmation }\end{array}$} & Cytology and imaging & 95 & $30 \%$ & \\
\hline & Cytology only & 12 & $4 \%$ & \\
\hline & Imaging only & 208 & $65 \%$ & \\
\hline & Clinical $^{*}$ & 3 & $1 \%$ & \\
\hline \multirow{4}{*}{ Sites of LM involvement } & Cranial only & 135 & $43 \%$ & 0.04 \\
\hline & Spine only & 84 & $26 \%$ & \\
\hline & Cranial and spine & 83 & $26 \%$ & \\
\hline & Missing & 16 & $5 \%$ & \\
\hline \multirow{2}{*}{$\begin{array}{l}\text { Non- CNS disease control } \\
\text { status }\end{array}$} & NED & 34 & $11 \%$ & 0.002 \\
\hline & Non-POD & 102 & $32 \%$ & \\
\hline
\end{tabular}

Clin Breast Cancer. Author manuscript; available in PMC 2018 February 01. 


\begin{tabular}{|c|c|c|c|c|}
\hline \multicolumn{2}{|l|}{ Characteristics } & \multirow{2}{*}{$\begin{array}{c}\mathbf{N} \\
168\end{array}$} & \multirow{2}{*}{$\begin{array}{c}\% \\
53 \%\end{array}$} & \multirow{2}{*}{$\begin{array}{l}\text { Univariate } \\
\text { association } \\
\text { with OS }\end{array}$} \\
\hline & POD & & & \\
\hline & Missing & 14 & $4 \%$ & \\
\hline \multirow{4}{*}{ Presence of brain metastases } & Prior to LMD diagnosis & 139 & $44 \%$ & \multirow[t]{4}{*}{0.007} \\
\hline & Concurrent with LMD diagnosis & 69 & $22 \%$ & \\
\hline & After LMD diagnosis & 8 & $3 \%$ & \\
\hline & No & 102 & $32 \%$ & \\
\hline \multirow{4}{*}{ LM specific treatment } & Radiation & \multicolumn{2}{|c|}{$20364 \%$} & \\
\hline & Intrathecal/ventricular therapy & 46 & $14 \%$ & \\
\hline & IV therapy (HD methotrexate) & 64 & $20 \%$ & \\
\hline & VP shunt placement & 60 & $19 \%$ & \\
\hline \multirow{2}{*}{$\begin{array}{l}\text { Systemic therapy use after LM } \\
\text { diagnosis }\end{array}$} & No & 126 & $40 \%$ & \multirow{2}{*}{$<0.001$} \\
\hline & Yes & 192 & $60 \%$ & \\
\hline
\end{tabular}

No information on imaging or cytology was available but patients were documented to have LM diagnosis and treated with LM specific treatment. Abbreviations: N, sample size, LMD, leptomeningeal disease; BC, breast cancer; MBC, metastatic breast cancer; KPS, Karnofsky Performance Status; CNS, central nervous system; HR, hormone receptor; NED, no evidence of disease; Non-POD, non-progressing disease; POD, progressive disease; IV, intravenous; HD, high dose; VP, ventriculoperitoneal 


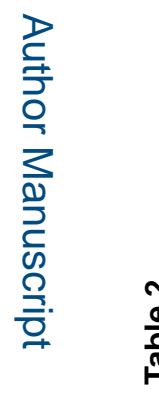

章

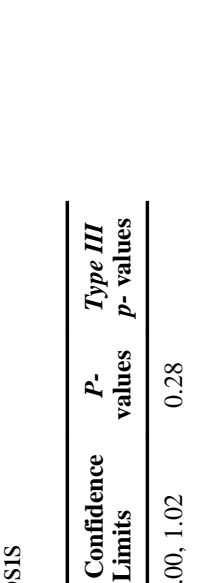

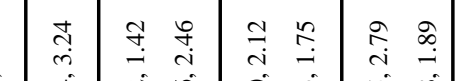

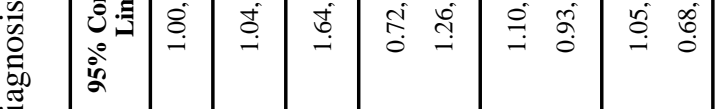

을

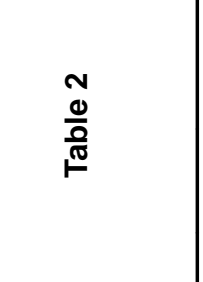

ग)

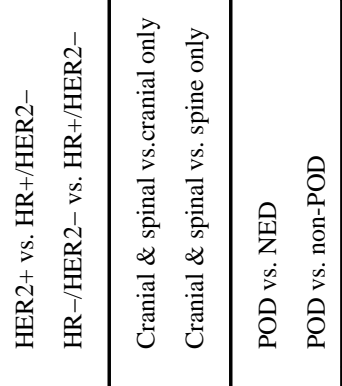

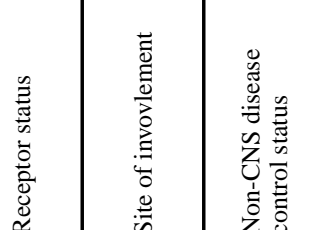

Clin Breast Cancer. Author manuscript; available in PMC 2018 February 01. 